\title{
Verpflichtung zu Open Access - universitäres Publizieren der Zukunft?
}

\author{
Fabienne Sarah Graf / Dario Henri Haux *
}

Unter Open Access versteht man den freien Zugang zu wissenschaftlicher Literatur. Sowohl der schweizerische Nationalfonds als auch Swissuniversities verlangen in immer verbindlicherer Form nach Open Access. Dabei stellt sich insbesondere die Frage, ob Universitätsangestellte zu Open Access verpflichtet werden dürfen. Die Autoren zeigen zunächst auf, wie diese Frage in anderen Ländern angegangen wurde und gehen danach auf eine mögliche Implementierung in der Schweiz ein.

I. Einleitung .230

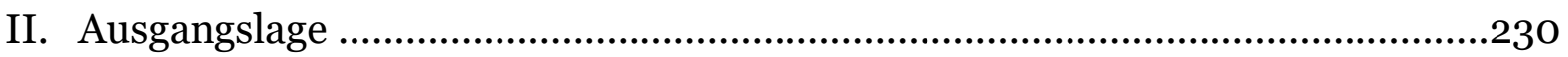

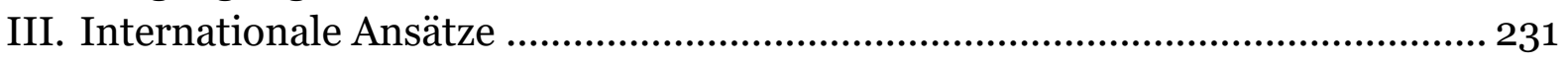

1. Harvard University ......................................................................... 231

2. University of Suffolk: UK Scholarly Communication License......................234

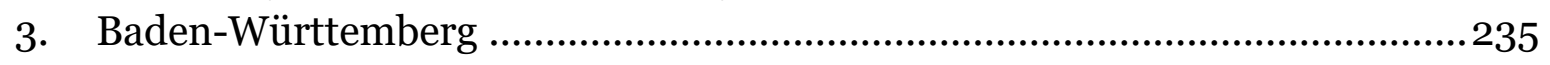

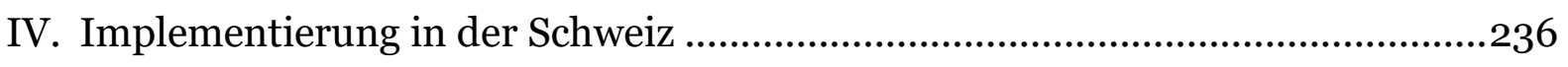

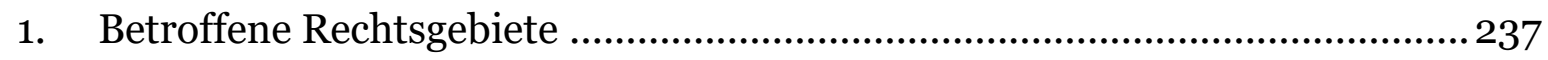

2. Das Beispiel der Universität St. Gallen und des Nationalfonds .................... 241

3. Herausforderungen für die Einführung von OA-Pflichten..........................242

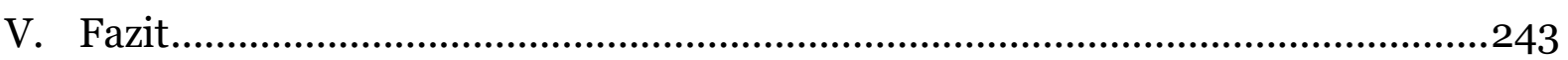

Zitiervorschlag: Fabienne Graf/Dario Haux, Verpflichtung zu Open Access universitäres Publizieren der Zukunft?, in: sui-generis 2017, S. 229

URL: $\quad$ sui-generis.ch/46

DOI: $\quad$ https://doi.org/10.21257/sg.46

* Fabienne Sarah Graf, Studentin der Rechtswissenschaft an der Universität Luzern, studentische Mitarbeiterin von Prof. Dr. Malte Gruber, Lehrstuhl für Rechtsphilosophie und Wirtschaftsrecht mit Schwerpunkt Immaterialgüterrecht und Recht der neuen Technologien, Universität Luzern. Dario Henri Haux, Jurist (1. Staatsexamen), wissenschaftlicher Assistent und Doktorand am oben genannten Lehrstuhl. Die Autoren danken Herrn Rechtsanwalt Dr. iur. Matthias Seemann sowie Herrn Prof. Dr. iur. Malte Gruber für ihre zahlreichen wertvollen Hinweise.

Dieses Werk ist lizenziert unter einer Creative Commons Namensnennung - Weitergabe unter gleichen Bedingungen 4.0 International Lizenz. 


\section{Einleitung}

1 In der Septemberausgabe der Schweizer Richterzeitung äusserte ein Richter Kritik am Parteiproporz und den damit zusammenhängenden Mandatssteuern. ${ }^{1}$ Werden in einem solchen Forum aktuelle Entwicklungen diskutiert, ist die Relevanz dieser Beiträge für fachliche Diskussionen und die Weiterentwicklung des Rechts unbestritten. Gleichzeitig sind diese Äusserungen, aufgrund des passwortgeschützten Abonnentenbereichs, grundsätzlich nur einem kleinen Personenkreis zugänglich. Sollten sie nicht offen zugänglich sein? Besteht Grund zum Handeln, um die Zugänglichkeit wissenschaftlicher Publikationen herzustellen? Insbesondere der auf die Annahme der nationalen Open Access (OA) -Strategie folgende Aktionsplan² setzt dieses Thema in der Schweiz auf die Agenda. Es sollen «passende Lösungen» aufgezeigt werden, um die mit privaten und öffentlichen Geldern finanzierten wissenschaftlichen Publikationen öffentlich zugänglich zu machen.3 Die Präsentation respektive Verabschiedung des Aktionsplans durch den Hochschulrat ist für Februar 2018 angesetzt.4 Die Zeit drängt und dennoch bleibt noch vieles ungeklärt. Eine der offenen Fragen, die das Verhältnis von Wissenschaftlerinnen, ihren Heiminstitutionen und Verlagen betrifft, ist Gegenstand dieses Beitrags: Dürfen und sollen Universitätsangestellte zu OA verpflichtet werden?

1 Giuliano Racioppi, Die moderne «Paulette»: Mandatssteuern von Richterinnen und Richtern, in: Justice - Justiz - Giustizia 2017/3.

2 Swissuniversities, Open Access.

3 Nationale Open-Access-Strategie für die Schweiz, 6. Implementierung, S. 6.

4 Swissuniversities, Open Access.

\section{Ausgangslage}

2 In den verschiedenen Fachrichtungen finden sich unterschiedliche OA-Ansätze, Modelle und Grundhaltungen.5 Während sich insbesondere in den naturwissenschaftlichen Disziplinen OA-Zeitschriften ein eigenes Renommee erarbeiten konnten, ${ }^{6}$ beherrschen in der Rechtswissenschaft weiterhin klassische Zeitschriftenverlage den Markt.7 Diese herausgehobene Stellung von Verlagen im Bereich analoger und digitaler Zeitschriftenabonnements hat in den vergangenen Jahren zu steigenden Kosten für die Bibliotheken geführt. ${ }^{8}$ Nur wenigen wird die Grössenordnung dieser Summen bekannt sein. 9

$5 \mathrm{Zu}$ den eklatanten Unterschieden zwischen Natur- und Geisteswissenschaften siehe Michael Link, Open Access im Wissenschaftsbereich, Diss. Erlangen-Nürnberg 2013, Frankfurt am Main 2013, S. 115; Daniel Hürlimann/Alexander Grossmann, Open Access als Utopie?, in: Informationspraxis 2017, S. 2.

6 Siehe die umfassende Initiative des Sponsoring Consortium for Open Access Publishing in Particle Physics oder auch Quantum. Für Nachwuchsforscher haben sich neue Publikationsmöglichkeiten etwa beim Emergent Scientist ergeben.

7 Hanjo Hamann, Freier Zugang zur juristischen Fachliteratur im Spiegel der Open-ScienceBewegung, in: sui-generis 2016, S. 96 ff., S. 102; Pflüger/Ertmann, E-Publishing und Open Access - Konsequenzen für das Urheberrecht im Hochschulbereich, in: ZUM 2004, S. 436 ff., S. 436; Eric W. Steinhauer, Das Recht auf Sichtbarkeit, Münster 2010, S. 17; kritisch Christian Russ, Freier Zugriff auf wissenschaftliche Beiträge?, in: ZRP 2004, S. 247 ff., S. 247, zu den möglichen Konsequenzen einer Systemänderung für die hergebrachte Zeitschriftenlandschaft.

8 Pflüger/Ertmann (Fn. 7), S. 437.

9 Eine Kostenübersicht findet sich etwa hier. Zum im Juni 2016 durch die Allianz der Wissenschaftsorganisationen in Deutschland getätigten Appell zur Offenlegung der Publikationsgebühren vgl. Thomas Hartmann, Eine juristische Agenda für digitale Inhalte, in: RBD 2016, S. 21 ff., S. 27; Die Frage ob ein hinreichendes öffentliches Interesse für die Einsichtnahme in die Verlagsverträge der Schweizer Hochschulbibliotheken mit Verlagshäusern bestehe, wurde vom Bundesgericht (Urteil 1C_4O/2017 vom 5. Juli 2017) verneint. Mit spezifischen Bezug auf die grundsätzlich in- 
Sinkende Bibliotheksbudgets führen da$\mathrm{zu}$, dass ein bedeutender Teil der wissenschaftlichen Literatur nicht mehr zugänglich ist. Mithin wächst das Interesse an OA, wobei aus der Sicht einer publizierenden Wissenschaftlerin theoretisch zwei Wege offenstehen. Zum einen die Veröffentlichung in einer Zeitschrift, welche Inhalte frei zugänglich online bereitstellt; betreten wird so der «goldene Weg».10 Zweitens, als sogenannter «grüner Weg», die Zugänglichmachung eines anderweitig veröffentlichten Beitrags in einem Online-Repositorium. ${ }^{11}$

3 Eine weitere Dimension bildet die Institutionalisierung der Idee von OA. Aktuelle Entwicklungen, etwa der erwähnte Ak-

formationsfreundliche Informations- und Datenschutzgesetzgesetzgebung des Kantons BaselStadt, erachteten die Richter es als «vertretbar, ernsthafte private und öffentliche Interessen von einem gewissen Gewicht für die Verweigerung des Zugangs zur nachgesuchten Information zu bejahen» (E. 6.2.2).

10 Eric Priest, Copyright and the Harvard Open Access Mandate, in: NJTIP 2012, S. 377 ff., S. $391 \mathrm{ff}$.

$11 \mathrm{Zu}$ den möglichen Organisationsmodellen von Repositorien siehe Margo Bargheer/Saskia Bellem/Birgit Schmidt, Open Access und Institutional Repositories - Rechtliche Rahmenbedingungen, S. 16 ff., in: Gerald Spindler (Hrsg.), Rechtliche Rahmenbedingungen von Open AccessPublikationen, Göttingen 2006, S. 1 ff.; zum Ganzen: Reto M. Hilty/Matthias Seemann, Open Access - Zugang zu wissenschaftlichen Publikationen im schweizerischen Recht, Rechtsgutachten im Auftrag der Universität Zürich, Zürich 2009, S. 16 ff.; Von OA-Anhängern wird der zweite Weg einer Veröffentlichung auf eigenen Websites («selfarchiving») vorgezogen., siehe Alma Swan, The Culture of Open Access: Researchers' Views and Responses, in: Neil Jacobs (Hrsg.), Open Access: Key Strategic, Technical and Economic Aspects, Oxford 2006, S. 65 ff., S. 71; vgl. auch Bargheer/Bellem/Schmidt (Fn. 11), S. 8. Zu den Repositorien siehe IUWIS Projekt, des Institut für Bibliotheks- und Informationswissenschaft an der Humboldt-Universität zu Berlin, Handreichung "Zur urheberrechtlichen Gestaltung von Repositorien" für Universitäten, Forschungszentren und andere Bildungseinrichtungen. tionsplan im Rahmen der nationalen OAStrategie, ${ }^{12}$ lassen die Thematik politisch erscheinen. ${ }^{13}$ Ziel ist gemäss der Vision 2024, dass zu diesem Zeitpunkt 100 Prozent der Publikationen von Schweizer Hochschulen OA veröffentlicht werden. ${ }^{14}$ Die Präsentation, respektive Verabschiedung durch den Hochschulrat ist für Februar 2018 angesetzt. 15

4 Solche Institutionalisierungsgedanken stehen im Spannungsfeld zwischen dem nationalen und internationalen Urheberrecht einerseits und der Idee von OA als globaler Wissenschaftskommunikation andererseits. ${ }^{16}$ In der Praxis wurden verschiedene, in ihren Besonderheiten geographisch beschränkte Ausprägungen der Institutionalisierung von OA, entwickelt. Einige von ihnen werden im Folgenden dargestellt.

\section{Internationale Ansätze}

\section{Harvard University}

5 Im Jahr 2008 stimmte die Faculty of Arts and Science der Harvard University ein-

12 Swissuniversities, Open Access, Kap. 1.

13 Vgl. Nicola von Lutterotti, Freier Zugang zur Wissenschaft, NZZ Online vom 3.6.2016.

14 Nationale Open-Access-Strategie, 4. Vision, S. 3.

15 Swissuniversities, Open Access: Als Ergebnis sind verschiedene Szenarien denkbar, welche von der Belassung der jetzigen Situation, über die Berücksichtigung der drei Grundoptionen («Offsetting Way», «Green Way», «Golden Way»), bis zu einer Kombination dieser reichen. Dazu: Nationale Open-Access-Strategie für die Schweiz, 5. Massnahmen, S. 4 f. In dieser Arbeit nicht angesprochen, aber doch wesentlicher Entscheidungsfaktor für oder gegen die einzelnen Szenarien sind die Kosten. Vertiefende wissenschaftliche Abhandlungen dazu wären wünschenswert.

16 Zur Begrenztheit des demokratiedeterminierten Handlungsrahmens siehe Thomas Hartmann, Open Access rechtlich absichern - warum es ein Opt-in braucht, in: Konstanze Söllner/Bernhard Mittermaier (Hrsg.), Praxishandbuch Open Access, Berlin 2017, S. 45 ff., S. 46. 
stimmig für die Abtretung des nicht ausschliesslichen Rechts ihrer Wissenschaftlerinnen auf sämtliche Formen nichtkommerzieller Nutzung von deren Werken an die Universität. ${ }^{17} \mathrm{Ihr}$ folgten andere Institutionen. ${ }^{18}$ Aus urheberrechtlicher Sicht handelt es sich bei dem genannten Modell um eine nichtexklusive Lizenz, ${ }^{19}$ wobei der Universität an grundsätzlich jedem Werk der eigenen Mitarbeiterinnen Rechte zufallen. ${ }^{20}$ Sobald ihr die finalen Werke vorliegen, werden sie im weltweit freien und ohne Registrierung zugänglichen DASH-Repositorium ${ }^{21}$ gespeichert. Eine nicht kommerzielle und nichtderivative Weiterverwendung durch Dritte ist möglich, muss aber den DASH-

17 Vgl. Robert Mitchell, Harvward to collect disseminate scholarly articles for faculty, Harvard Gazette vom 14. Februar 2008; Priest (Fn. 10), S. 381, S. 396. Die Umsetzung basiert auf einem Ansatz des US National Institute of Health (NIH) von 2005, dazu Orit Fischmann Afori, The Battle Over Public E-Libraries - Taking Stock and Moving Ahead, in: IIC 2013, S. 392 ff., S. 398; Priest (Fn. 10), S. 398.

18 Eine Aufzählung der Organisationen findet sich bei Priest (Fn. 10), S. 396. Zum Einfluss der herausragenden Stellung dieser Ivy LeagueUniversität in diesem Prozess siehe Peter Suber, The Open Access Mandate at Harvard, in: SPARC Open Access Newsletter Nr. 119 2008; Philip Young, The Serials Crisis and Open Access, A White Paper for the Virginia Tech Commission on Research, Virginia Polytechnic Institute and State University 2009.

19 Priest (Fn. 10), S. 381. Siehe als FAQ zum Harvard-Modell auch die sehr übersichtliche Seite der UK Publishers Association.

20 Vgl. Ellen Finnie Duranceau/Sue Kriegsman, Implementing Open Access Policies Using Institutional Repositories, in: Pamela Bluh/Cindy Hepfer (Hrsg.), The institutional repository: Benefits and Challenges, Chicago 2013, S. 75 ff., S. 79, «Grundsätzlich» da Ausstiegsmöglichkeiten bestehen.

21 Digital Access to Scholarship at Harvard. Es entstand aus der Zusammenarbeit zwischen dem Office for Scholarly Communication (OSC) und dem Office for Information Systems (OIS), zwei Programme der Harvard Universitätsbibliothek, dazu Peter Kosewski, Harvard's DASH for open access, Harvard Gazette vom 31. August 2009.
Konditionen entsprechen. ${ }^{22}$ Grundsätzlich versuchen die Universitäten sich schriftliche Lizenzen einräumen zu lassen.23 Eine Speicherung im DASHRepositorium kommt damit als, mitunter simultane Erstveröffentlichung einer Golden Way-Publikation in einem anderen Medium gleich. ${ }^{24}$

6 Vorgesehen bleiben Freistellungen von einer Pflicht zu OA, sogenannte «waiver», sowie die Respektierung von allfälligen Embargo-Fristen durch das Repositorium. ${ }^{25}$ Doch wie kann eine an der Universität angestellte Forscherin von der erwähnten Sonderfreistellung (Opt-OutMöglichkeit) Gebrauch machen? Diese wird beim Dekan der Fakultät beantragt, z.T. mit Begründung versehen, und grundsätzlich genehmigt. ${ }^{26}$ Gleichwohl werden nur in etwa $5 \%$ der Fälle solche Anträge überhaupt gestellt. ${ }^{27}$ Eine zweite Ausnahme von der OA-Pflicht stellt die sogenannte «dark deposition» dar. In

$22 \overline{\text { Die Wortwahl ähnelt derjenigen der Budapest }}$ Initiative.

23 Michael W. Carroll, Complying with the NIH Public Access Policy, Considerations and Options, SPARC 2008; Priest (Fn. 10), S. 432.

24 Axel Metzger, Die urheberrechtliche Gestaltung von Open Access Repositorien, Gutachten, in: IUWIS Handreichung (Fn. 11), S. 55.

25 Siehe dazu den Wortlaut der OA-Policy der Harvard Faculty of Arts and Sciences; vgl. Priest (Fn. 10), S. 381 ff., 397 f. Gesprochen wird auch von "opt out". In der Praxis werden in weniger als $5 \%$ der Fälle «waiver» beantragt, dazu: Chris Banks, Focusing upstream: supporting scholarly communication by academics, in: UKSG Insights 29 2016, S. 37 ff., S. 42; vgl. Duranceau/Kriegsman (Fn. 20), S. 79.

26 Begründungspflicht am Beispiel der OA-Policy der Harvard Law School: «The Dean or the Dean's designate will waive application of the policy to a particular article upon written request by a Faculty member explaining the need.»

27 Stuart Shieber/Peter Suber, Good Practices For University Open-Access Policies (2013), Cambridge, MA: Harvard, Open Access Project; Young (Fn. 18), S. $7 \mathrm{f}$. 
diesem Fall lassen es vertragsrechtliche Bestimmungen zwischen der Wissenschaftlerin als Autorin und dem Verlag nicht zu, dass das Werk simultan auch im Repositorium zugänglich gemacht wird. Nach Ablauf eines etwa 6-monatigen Embargos, dem sogenannten «lift date», erfolgt dann die Öffnung des Werkes für die Allgemeinheit. ${ }^{28}$

7 Trotz des anhaltenden Erfolgs des Modells, werden auch weiterhin Diskussionen zur Frage des Eingriffs in die Wissenschaftsfreiheit geführt. Die Forscherin könne nicht mehr entscheiden wo, wann und was sie veröffentliche. ${ }^{29}$ Eine weitere, mitunter technische Herausforderung bildet die Klassifizierung der verschiedenen Versionen. In den Datenbanken wird zwischen Autor-Manuskript, Verlagsversion und anderen unterschieden. ${ }^{30}$ Die Unterscheidung ist von zentraler Bedeutung, untersagen doch etliche Verlage die Distribution der «version of record», der finalen Verlagsversion. ${ }^{31}$ Unklar bleiben

28 Harvard Library, Office for Scholarly Communication, Author Frequently Asked Questions.

29 Zum Diskussionsstand in Deutschland: Matthias Mönch/Jens. M. Nödler, Hochschulen und Urheberrecht - Schutz wissenschaftlicher Werke, in: Gerald Spindler (Hrsg.), Rechtliche Rahmenbedingungen von Open Access-Publikationen, Göttingen 2006, S. 21 ff., S. 40; Pflüger/Ertmann (Fn. 7), S. 441; Arthur-Axel Wandtke/Winifired Bullinger, Praxiskommentar zum Urheberrecht, $\S 43$ UrhG, 4. Aufl., München 2014, Rn. 40-43.

30 Relevant ist hier insb. die «version of record» als finale Version eines Verlages, in Abgrenzung zu verschiedenen Autor-Manuskripten, dazu Daniel Rothchild/Stuart Shieber, Automatically Determining Versions of Scholarly Articles, in: SRC 2017, S. 2; vgl. Melanie Bosshart, Das CreativeCommons-Lizenzsystem, Diss., Zürich 2013, S. 227.

31 Vorgeschlagen wird ein automatisierter Prüfvorgang unter Verwendung von Algorithmen des maschinellen Lernens (logistische Regression), welche sich im Direktvergleich mit der manuellen Prüfung durch Experten bereits bewäh-ren konnte. Zum Ganzen Rothchild/Shieber (Fn. 30), auch die Kriterien der «waiver»Genehmigung oder Ablehnung. Während einige Fakultäten umfassende Begründungen verlangen, ergehen anderorts Genehmigungen ohne jegliche Rückfrage. $3^{2}$ Bezogen auf die konkrete Situation in den USA ist ferner fraglich, inwiefern die gesetzliche «work made for hire»Regelung33 dazu führen könnte, dass gar nicht die Wissenschaftlerin selbst, sondern die Universität als Arbeitgeberin, als Urheberin anzusehen wäre. 34

8 Dennoch erscheint das Harvard-Modell als sinnvolle Ergänzung zum gesetzlichen US-Copyright-System. Ein Grund liegt darin, dass dieses allein - trotz Limitationen und Ausnahmen zugunsten von Bildung, Wissenschaft und Archivierung - dem vollen Potential der neuen Technologien für die Bildungsinstitutionen nicht gerecht zu werden vermag. 35

S. 1 ff.; vgl. Duranceau/Kriegsman (Fn. 20), S. 81.

32 Kritisch dazu Karin Wulf/Simon Newman, Missing the Target: The UK Scholarly Communications License, in: The Scholarly Kitchen 2017. Diese sehen in der «waiver»-Option einen möglichen Weg der Umgehung des Systems - und in diesem «more of a statement of institutional preference than a directive».

3317 U.S. Code § 101; vgl. Inge Hochreutener, Der Verlagsvertrag, Art. 380-393 OR, in: Jörg Schmid, Kommentar zum schweizerischen Zivilrecht, 4. Aufl., Zürich 2011, S. 20.

34 Kritisch dazu Priest (Fn. 10), S. 400 ff. Denkbar ist dieses Szenario insb. da das US CopyrightSystem (anders als das kontinentaleuropäische Recht) kein Urheberpersönlichkeitsrecht im eigentlichen Sinne kennt und daher an die Rechtsnutzung anknüpft, dazu: Hochreutener (Fn. 33), S. 20.

35 Vgl. Philipp Usadel, Copyright law and the access to education and knowledge in the digital age: matching limitations and exceptions in Portugal, Brazil and Mozambique, Aachen 2016, S. 172 f. Zum Einfluss der Prominenz dieser Ivy-LeagueUniversität: siehe Suber (Fn. 18). Zum USCopyright System: Lawrence Lessig, The Creative Commons, Montana Law Review, 2007 65, S. $4 \mathrm{ff}$. 


\section{University of Suffolk: UK Scholarly Communication License (UK-SCL)}

9 Auch in Grossbritannien haben sich vielfältige OA-Ansätze und institutionelle Regelwerke entwickelt.36 Dabei weisen einige Universitäten schon jetzt einen Etat für Golden Way-Publikationen aus. In diesem Umfeld werden OA-Ansätze institutionsübergreifend diskutiert 37 und sind vermehrt auch breit abgestützt. ${ }^{8}$ An der University of Sussex 39 wird etwa ein auf dem Harvard-Modell basierender Ansatz verfolgt.40 Neben der Zugänglichmachung über die Einrichtung einer Datenbank wird Wert auf eine rechtswirksame Nachnutzung der hinterlegten Werke gelegt.41 Dies geschieht mithilfe standardisierter «Creative Commons (CC)-Lizenzen», zur Ermöglichung der Nutzung des Beitrags durch Dritte. $4^{2}$ Der Urheber entscheidet in diesem Kontext über den Umfang der den Nutzern der OA-Publikation eingeräumten Rechte.43

$36 \overline{\text { Elizabeth Gadd, UK university policy approaches }}$ towards the copyright ownership of scholarly works and the future of open access, in: Aslib Journal of Information Management 2017, S. 95 ff., S. 97.

37 Banks (Fn. 25), S. 39 f. Vgl. dazu auch den Finch report 2012 (Bericht der «Working Group on Expanding Access to Published Research Findings») und die Antwort des BIS (UK Department for Business Innovation and Skills) vom 16.7.2012.

38 UK Scholarly Communications Licence, University of Suffolk, S. 8.

39 Vgl. UK Scholarly Communications Licence, University of Sussex (Fn. 38), S. 1. Auch hier (gleich wie unter dem Harvard-Modell) entsteht die Verpflichtung zur OA-Publikation universitätsintern.

40 Die Universität stellt selber eine ModellRichtlinie für die Adaption durch andere Institutionen bereit. Leitende Funktion in der Erarbeitung hat insb. das Imperial College London, dazu Gadd (Fn. 36), S. 97 f.

41 Hartmann (Fn. 16), Opt-in, S. 48.

42 UK Scholarly Communications Licence, University of Suffolk (Fn. 38), S. 5; vgl. Hartmann (Fn. 16), Opt-in, S. 48.

43 Michael Fehling, Von der kommerziellen Verlagsproduktion zum Open Access - Zur Rolle des Rechts beim Wandel wissenschaftlicher Publika-
Befürworter dieser von CC-Lizenzen betonen, dass auf diese Weise den Bedürfnissen der Urheber besser entsprochen werde, als dies im tradierten, auf Belohnung basierenden System der Fall ist.44 Mit der Anwendung der CC-Lizenzen unterscheidet sich dieses Modell vom Harvard-Modell. Gleichwohl ist gemäss den DASH-Bedingungen auch dort eine Nachnutzung möglich.

10 An der University of Suffolk findet unter der «UK Scholarly Communications Licence» die «CC-BY-NC»-Grundform der CC-Lizenz Anwendung.45 Diese umfasst die Module der «Namensnennung» («BY») und der «nicht kommerziell(en)» («NC») Nutzung. Mögliche Erweiterungen ergeben sich durch die Module der «Weitergabe unter gleichen Bedingungen» («SA») und «keine Bearbeitung» («ND»).46 Notwendige Voraussetzung jeder Unterform der Nutzung bildet mithin die Namensnennung, d.h. die Zurechnung der Publikation zum Autor. 47

11 Die University of Suffolk wendet zudem das Konzept der «moving wall»-Lizenz an. Dabei werden mit der Repositoriumshinterlegung die Metadaten ersichtlich gemacht. 48 Auch bei der UK-SCL

tionskanäle, in: Wolfgang Hoffmann-Riem (Hrsg.), Innovationen im Recht, Baden-Baden 2016, S. 337 ff., S. 342; Lessig (Fn. 35), S.11.

44 Bosshart (Fn. 30), S. 63. Die Belohnung ergäbe sich in der Förderung der Werkschöpfung. Zur Vereinbarkeit der Lizenzierung mit dem nationalen Recht: vgl. Miriam Sahlfeld, Creative Commons, in: Medialex 2007, S. 72 ff., S. 74 ff.

45 Am Beispiel der Universität Suffolk: UK Scholarly Communications Licence, University of Suffolk (Fn. 38), S. 5.

46 Bosshart (Fn. 30), S. 64 f.; vgl. Lessig (Fn. 35), S. 11.

47 Stuart M. Shieber, The Case for the Journal's Use of a CC-BY License, in: JLM 2012, S. 5 ff., S. 6.

48 Banks (Fn. 25), S. 42. Damit wird auch den OAKriterien der grossen europäischen Fördergefässe 
sind Sonderfreistellungen vorgesehen. Anders als beim Harvard-Ansatz ist dabei jedoch mehr Spielraum für die Institutionen gegeben, innerhalb dessen Konditionen für die Verweigerung der «waiver» festgelegt werden können.49 Auch dieses Modell folgt der Annahme, dass das Urheberrecht an einem Werk bei dessen Autor und nicht bei der Universität entsteht.50

\section{Baden-Württemberg}

12 In Deutschland wurde die OA-Idee von der Allianz der Wissenschaftsorganisationen ${ }^{1}$ wie auch, zum Erstaunen vieler, vom Rechtsausschuss des Bundestags vorangetrieben..$^{2}$ Durch den am 1. Januar 2014 eingefügten und in Kraft getretenen

entsprochen (vgl. z.B. für die ERC [European Research Council] Grants); vgl. Duranceau/Kriegsman (Fn. 20), S. 87.

49 Gadd (Fn. 36), S. 98.

50 Siehe Kap. III.1; Gadd (Fn. 36), S. 98. Vgl. dazu den CDPA (Copyright Designs and Patents Act, 1988). Darin: «Where a literary, dramatic, musical or artistic work, or a film, is made by an employee in the course of his employment, his employer is the first owner of any copyright in the work subject to any agreement to the contrary»; vgl. Hochreutener (Fn. 33), S. 20 f.; Zur tatsächlichen Erhebung von Urheberrechtsansprüchen in Richtlinien von UK-Universitäten: siehe Gadd (Fn. 36), S. 105 ff., 111. Die Autorin sieht in der unterbliebenen Klärung der kontroversen Urheberrechtsfrage gar die Ursache für die Entwicklung des Gefüges von OA-Ansätzen und in der gemeinsamen Urheberschaft die Möglichkeit zur Auflösung verschiedener OA-Problemfelder.

51 Vgl. dazu die Berliner Erklärung vom 22. Oktober 2003 als Grundstein; Marcus Hirschfelder, Anforderungen an eine rechtliche Verankerung des Open Access Prinzips, Diss. Saarbrücken 2007, S. $10 \mathrm{ff}$.

${ }^{2}$ Vgl. Entschliessung vom 26.6.2013, S. 2 ff.; Fehling (Fn. 43), S. 340. $\mathrm{Zu}$ den Vorgaben des EURechts: vgl. Daniel Krausnick, Offene Wissenschaft? - Öffentlich-rechtliche Aspekte der Diskussion um Open Access und Open Data, in: Max-Emanuel Geis/Markus Winkler/Christian Bickenbach (Hrsg.), Von der Kultur der Verfassung, Festschrift für Friedhelm Hufen, München 2015, S. 367 ff., S. $371 \mathrm{ff}$.
$\S 38$ Abs. 4 des deutschen Urheberrechtsgesetzes (UrhG), wurde Wissenschaftlerinnen, welche ihre Arbeit mit überwiegend staatlich finanzierten Drittmitteln finanzieren, bundesweit ein sich - nach einer vertraglich nicht abdingbaren Karenzzeit von einem Jahr aktivierendes Zweitveröffentlichungsrecht53 gesichert.54 In der Gesetzesbegründung55 des deutschen Gesetzgeber werden erstmals deutlich "zentrale Begründungen des Open-AccessParadigmas" aufgegriffen. $5^{6} 2017$ hat die Bundesregierung sodann den "Entwurf für ein Gesetz zur Angleichung des Urheberrechts an die aktuellen Erfordernisse der Wissensgesellschaft (UrhWissG)" beschlossen. 57

13 Speziell ausgeprägt ist die OAVerpflichtung seit dem dritten Hochschulrechtsänderungsgesetz vom 1. April 2014 (3. HRÄG) und mit Erlass des § 44 Abs. 6 Landeshochschulgesetz (LHG) in Baden-Württemberg. Durch Satzung

53 Zur umstrittenen Klassifikation dieses Rechts als (europarechtswidrige) Schrankenregelung im Vorfeld der Revision: Hirschfelder (Fn. 51), S. 138 f.; a.M. Gerd Hansen, Für ein Zweitveröffentlichungsrecht für Wissenschaftler - zugleich Besprechung von Marcus Hirschfelder: Anforderungen an eine rechtliche Verankerung des Open Access Prinzips, in GRUR Int. 2009, S. 799 ff., S. $802 \mathrm{ff}$.

54 Ulrike Müßig, "Ein Knauf als Tür": Open AccessVerpflichtung durch Forschungsförderung vs. Gemeinfreiheitsgrenzen digitaler Wissenschaftskommunikation, in: JZ 2015, S. 221 ff., S. 221, 229.

55 Deutscher Bundestag, Drucksache 17/13423 vom 8.5.2013, S. 9 f.

56 Hartmann (Fn. 16), Opt-in, S. 48. Was fortschrittlich anmutet, wird aber auch begleitet von rechtlichen Bedenken: dazu u.a. Müßig (Fn. 54), S. $229 \mathrm{ff}$.

57 Deutscher Bundestag, Drucksache 18/12329 vom 15.5.2017; Katharina De la Durantaye, Neues Urheberrecht für Bildung und Wissenschaft - eine kritische Würdigung des Gesetzesentwurfs, in: GRUR 2017, S. $558 \mathrm{ff}$. 
werden Wissenschaftlerinnen verpflichtet, das Zweitveröffentlichungsrecht aus $\S 38$ Abs. 4 UrhG in Anspruch zu nehmen..$^{8}$ Damit besteht eine dienstrechtliche Absicherung. Das Recht wird zur Pflicht.59 Dabei stellt sich die Frage nach der Verfassungskonformität einer solchen Pflicht.60 Das Bundesverfassungsgericht stellt schon die Entscheidung der Wissenschaftlerin über eine Publikation unter das Grundrecht der Forschungsfreiheit (Art. 5 Abs. 3 Grundgesetz, GG). ${ }^{61}$ Neben der sich daraus ergebenden Verfassungswidrigkeit widerspricht die Zweitveröffentlichungspflicht auch $\S 3$ Abs. 2 LHG, wo die Forschungsfreiheit ebenfalls verankert ist. ${ }^{62}$ Neben diesen inhaltlichen Aspekten kommt die Frage hinzu, ob den Bundesländern die Gesetzgebungskompetenz zusteht. Dies muss

${ }_{58} \overline{\text { Manfred Löwisch, Förderung statt Zwang - Neue }}$ Open Access Strategie in Baden-Württemberg, in OdW 2017, S. 59 f.; Georg Sandberger, Landeshochschulgesetz Baden-Württemberg, Kommentar, 2. Aufl., Heidelberg 2015, S. 21. Die Anordnung zur Einstellung der Publikation in ein Repositorium erfolge zuvor durch eine Rechtsverordnung, nun durch eine Satzung der Hochschule. Die Frist beträgt $1 \mathrm{Jahr}$ und ist somit an $\S 38$ Abs. 4 UrhG angepasst (im Anhörungsentwurf waren noch 6 Monate vorgesehen), dazu: Krausnick (Fn. 52), S. 378.

59 Christian von Coelln/Volker M. Haug (Hrsg.), BeckOK Hochschulrecht Baden-Württemberg, Rn. 20 zu § 44 LHG; Sandberger (Fn. 58), S. 40.

60 Zentral ist die Unterscheidung von Zweitveröffentlichungsrecht und -pflicht: dazu Sandberger (Fn. 58), S. 70; vgl. Coelln/Haug (Fn. 59), Rn. 21 $\mathrm{zu} \S 44$ LHG.

61 BVerfG, Beschluss vom 1.3.1978, BVerfGE 47, 327, 367; Beschluss vom 11.1.1994, BVerfGE 90, 1, 12; BGH Urteil vom 27.9.1990, BGHZ 112, 243 ff.; vgl. Müßig (Fn. 54), S. 231 f.; Krausnick (Fn. 52), S. 378.

62 «Die Freiheit der Forschung (Artikel 5 Absatz 3 Satz 1 GG) umfasst insbesondere die Fragestellung, die Grundsätze der Methodik sowie die Bewertung des Forschungsergebnisses und seine Verbreitung. Satz 1 gilt für künstlerische Entwicklungsvorhaben und für die Kunstausübung entsprechend.» (§ 3 Abs. 2 LHG); Sandberger (Fn. 58), S. 40. verneint werden, sofern in der Bestimmung eine Schranke des Urheberrechts und somit materielles Urheberrecht erblickt wird. 63 Ergibt sich damit aus dem LHG eine Beschwer einzelner Wissenschaftlerinnen? Das Gesetz richtet die Satzungspflicht an die Universtäten, also nicht direkt an Einzelne.64 Das Gelingen der Einführung einer OA-Verpflichtung, genauso wie das Vorgehen gegen eine solche, hängt somit letztlich von den jeweiligen Satzungen der Universitäten und ihrer Umsetzung ab. 65

\section{Implementierung in der Schweiz}

14 Die Frage, ob Universitätsangestellte zu OA verpflichtet werden dürfen oder sollen, kann nicht allein aus dem jeweiligen Universitätsrecht heraus beantwortet werden. Vielmehr müssen die bisherigen Rechtsentwicklungen, Umsetzungsansätze und künftigen Herausforderungen in die Abwägung mit einfliessen. ${ }^{66}$ Dabei ist

63 Zur Sperrung einer Landeskompetenz: Art. 73 Abs. 1 Nr. 9 GG i.V.m. Art. 71 GG; gl.M. Krausnick (Fn. 52), S. 378.

64 Sodann müsste eine Klage gegen die Gesetzesbestimmung durch die Universität an die Hand genommen werden; das wiederum erfordert eine breite Unterstützung über die Fakultätsgrenzen hinweg, diese dürfte aber an den verschiedenen Einstelllungen zu OA (siehe Kap. II) und politischen Ausrichtungen in den Fächern, scheitern.

65 Die erste Normkontrolle wurde von 17 Konstanzer Professoren am Verwaltungsgerichtshof Baden-Württemberg anhängig gemacht. Dieser hat mit Beschluss vom 26. September 2017 dem Bundesverfassungsgericht die Frage vorgelegt, ob $\S 44$ Abs. 6 des Landeshochschulgesetzes gegen Art. 71, Art. 73 Abs. 1 Nr. 9 GG verstösst. (vgl. Medienmitteilung des Verwaltungsgerichtshof Baden-Württemberg vom 6. November 2017 sowie die Berichterstattung bei lto.de). Da andere Universitäten planen, ihr Vorgehen von dieser Entscheidung abhängig zu machen, kommt diesem Verfahren eine nicht zu unterschätzende Bedeutung zu, siehe dazu den Artikel in der Badischen Zeitung: Sollen Wissenschaftstexte im Internet frei zugänglich werden?.

66 Aktualisiert wurde diese Kalkulation mit der Ver- 
insbesondere nicht $\mathrm{zu}$ vergessen, dass neben der Konsultation von kantonalem Recht, aufgrund der Trägerschaft von Universitäten 67 im Bundesrecht, auch das Hochschulförderungs- und koordinationsgesetz (HFKG, 68 vgl. dessen Art. 1 Abs. 1) und das Universitätsförderungsgesetz (UFG) ${ }^{69}$ zu berücksichtigen sind. 70

\section{Betroffene Rechtsgebiete}

15 Open Access und eine damit allfällig verbundene Verpflichtung betrifft folglich vielfältige Rechtsgrundlagen. Im Folgenden soll insbesondere auf verfassungs-, urheberrechtliche- und wettbewerbsrechtliche Aspekte eingegangen werden. Unterschieden wird dabei zwischen der Perspektive der Universität und der ihrer wissenschaftlichen Angestellten.

\section{a) Verfassungsrecht}

16 Aus verfassungsrechtlicher Sicht ist zunächst zu fragen, ob eine $\mathrm{OA}$ Verpflichtung auf die Wissenschaftsfreiheit nach Art. 27 BV gestützt werden

abschiedung der nationalen OA-Strategie durch die Plenarversammlung von swissuniversities am 31.Januar.2017 und somit der unmittelbar bevorstehenden tatsächlichen Implementierung in der Schweiz. Vgl. dazu, mit Zeitplan: Swissuniversities, Open Access.

67 Siehe BV 63a, UFG 1 I, ZSAV: Grds. kantonal, Ausnahmen bilden die ETHZ und die EPFL.

68 Bundesgesetz über die Förderung der Hochschulen und die Koordination im schweizerischen Hochschulbereich (Hochschulförderungs- und -koordinationsgesetz, HFKG), vom 30. September 2011 (Stand 1. Februar 2017).

69 Bundesgesetz über die Förderung der Universitäten und über die Zusammenarbeit im Hochschulbereich (UFG) vom 8. Oktober 1999 (Stand 1.1.2013).

70 Vgl. Fabian Amschwand, Qualitätssicherung im schweizerischen Hochschulwesen Verfassungsrechtlicher Rahmen und Leitlinien für die Umsetzung an den Hochschulen, in: Heinz Hausheer, ASR - Abhandlungen zum Schweizerischen Recht, Nr. 802, Bern 2014, S. 15 ff., S. 23 f. könnte. ${ }^{11}$ Die Wissenschaft72 setzt, als Teil des Meinungsbildungsprozesses, in einem besonders hohen Mass eine institutionelle Einbettung voraus.73 Für die Universitäten bedeutet das, als Voraussetzung für die Unabhängigkeit der Wissenschaftlerinnen, organisatorische $\mathrm{Au}-$ tonomie.74 Daraus richtet sich eine programmatische Weisung an den Staat die wissenschaftliche Forschung und die Innovation zu fördern (vgl. Art. 64 Abs. 1 BV). 75 Inhaltlich bedarf es der Klärung, inwiefern die Wissenschaftsfreiheit mit dem OA-Gedanken kompatibel ist.

17 Grundsätzlich erscheint die Wissenschaftsfreiheit OA-freundlich. Einem

$71 \overline{\text { Diese Frage stellt sich sowohl im Zusammenhang }}$ mit dem Urheberrecht, dem öffentlichen Dienstrecht und dem Hochschulrecht. Sie wird daher als zentrale Vorfrage betrachtet. Vgl. dazu; Steinhauer (Fn. 7), S. 45.

72 Der Begriff «Wissenschaft» wird in der Schweizer Rechtsordnung nicht definiert, von den Materialen zur BV jedoch vorausgesetzt und vom BG nicht weiter erörtert. Dazu Rainer J. Schweizer/Felix Hafner, Art. 20 BV, in: Die Schweizerische Bundesverfassung, 4. Aufl., Zürich/St. Gallen 2014, Rn. 8 zu Art. 20; vgl. Giovanni Biaggini, BV Kommentar, Zürich 2007, S. 163 f.

73 Jörg Paul Müller/Markus Schefer, Grundrechte in der Schweiz, 4. Aufl., Bern 2008, S. 544 ff. Diesen Rahmen zur Entfaltung benötigt auch die Wissenschaftsfreiheit, dazu: Biaggini (Fn. 72), S. 163.

74 Müller/Schefer (Fn. 73), S. 544. Vgl. zum Aspekt der Unabhängigkeit, auch bei staatlicher Trägerschaft der Universität: Regina Kiener/Walter Kälin, Grundrechte, 2. Aufl., Bern 2013, S. 268.

75 Vgl. Paul Richli, Von der Gelehrtenrepublik zur Managementuniversität?: Rechtsfragen der Organisation und Leitung von Universitäten in der Bundesrepublik Deutschland, Österreich, der Schweiz und den USA, Bern 2009, S. 149. Diese Pflicht erstreckt sich, als sekundäres Schutz-ziel, auch auf die Wirtschaftsfreiheit (vgl. Art. 27 und 94 BV) i.S. der Notwendigkeit wettbewerbsneutralen Verhaltens, dazu: Markus Müller, Akademische Freiheit, Sorgen um ein bedrohtes Gut, in: Berner Gedanken zum Recht : Festgabe der Rechtswissenschaftlichen Fakultät der Universität Bern für den Schweizerischen Juristentag 2014, Bern 2014, S. 381 ff., 388 f. 
Bild der Wissenschaft, deren Hauptfunktion der auf entsprechenden Methoden basierende Erkenntnisgewinn ist, 76 kommt das Ziel der freien Zugänglichkeit von wissenschaftlichen Informationen inhaltlich zu Gute. 77 Denn Teil der Wissenschaft ist auch «deren Verbreitung in einem offenen, rationalen Diskurs».78 Freies Publizieren bedeutet, neben der Bestimmung des jeweiligen Werks zur Verbreitung, auch die Option zur Wahl des Zeitpunkts und des Kanals.79 Dieser Aspekt zeigt, dass eine Verpflichtung zur OA-Publikation zur Wissenschaftsfreiheit in einem Spannungsverhältnis steht. Entsprechend ist eine solche Verpflichtung von vornherein nur unter Berücksichtigung von Verhältnismässigkeitserwägungen möglich. 80

Nicht nur die Forschenden als natürliche Personen, sondern auch juristische Personen können Grundrechtsträger von Art. 27 BV sein. In der Praxis ungeklärt ist die Frage, inwiefern auch Hochschulen miteinzubeziehen sind. ${ }^{81}$ Die Wissenschaftsfreiheit gilt, im Zuge der indirek-

76 Vgl. Kiener/Kälin (Fn. 74), S. 266. Dabei darf in einem demokratischen Verfassungsstaat kein einzelnes Wissenschaftsverständnis den Vorzug erhalten. Vielmehr muss deren Pluralität den Begriff und somit die Schutzobjekte definieren, dazu Kiener/Kälin (Fn. 74), S. 268; vgl. Müller/ Schefer (Fn. 73), S. 543.

77 Siehe Kap. II; vgl. Nicole Schmidt, Open Access, Hochschulrechtliche Veröffentlichungs- und urheberrechtliche Anbietungspflichten des Hochschulprofessors, Diss. Dresden 2015, BadenBaden 2016, S. 19, 47.

78 Schweizer/Hafner (Fn. 72), Rn. 8 zu Art. 20; vgl. Kiener/Kälin (Fn. 74), S. 267.

79 Vgl. Biaggini (Fn. 72), S. 163; Schweizer/Hafner (Fn. 72), Rn. 22 zu Art. 20.

80 Eine Einschränkung von Art. $20 \mathrm{BV}$ muss sich an Art. 36 BV messen lassen: Biaggini (Fn. 72), S. 164.

81 Biaggini (Fn. 72), S. 164. I.S. der Autonomie wird dies durch die Lehre bejaht; u.a. Kiener/Kälin (Fn. 74), S. 266; vgl. Schweizer/Hafner (Fn. 72), Rn. 22 zu Art. 20. ten Drittwirkung der Grundrechte, nicht nur gegenüber dem Staat, sondern muss auch bei der Rechtsauslegung gegenüber Privaten beachtet werden. ${ }^{82}$ Im Lichte einer OA-Verpflichtung erscheint privatrechtlich primär die Auslegung des Urheberrechtsgesetzes (URG) relevant. Ob und inwiefern einer grundrechtskonformen Auslegung auch in der Praxis Gewicht zukommt, ist streitig. ${ }^{83}$ Dabei schränkt der Sinn und Zweck der Norm eine zu weitreichende Auslegung durchaus ein. ${ }^{84}$ Zum anderen ist eine Auslegung nicht schon BV-konform, wenn sie der Wissenschaftsfreiheit entspricht; vielmehr fliessen auch die anderen Verfassungsbestimmungen mit ein. 85 Neben der Wissenschaftsfreiheit wird hier auch die Eigentumsgarantie angeführt. ${ }^{86}$ So gelten auch Immaterialgüterrechte als Schutzobjekte der Eigentumsgarantie gemäss Art. 26 BV.87 Interessant ist dabei, dass sich in den (Grund-)Freiheiten und dem Urheberrecht diametral verschiedene Interessen widerspiegeln. Ein Umstand, welcher durch die Debatten des digitalen Zeitalters verstärkt Einzug in das öffentliche Bewusstsein findet. 88

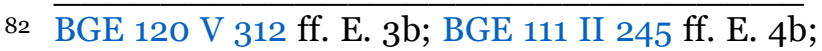
vgl. BGE 136 I 167 ff. E. 2.2.

83 Abgesprochen wird die Bedeutung durch Hilty/Seemann (Fn. 11), S. 22.

84 Vgl. Thomas Gächter, Rechtsmissbrauch im öffentlichen Recht, Unter besonderer Berücksichtigung des Bundessozialversicherungssrechts. Ein Beitrag zu Treu und Glauben, Methodik und Gesetzeskorrektur im öffentlichen Recht, Zürich 2005, S. 280 f. Zustimmend Hilty/Seemann (Fn. 11), S. 22.

85 Schweizer/Hafner (Fn. 72), Rn. 8 zu Art. 20.

86 Hilty/Seemann (Fn. 11), S. 22; vgl. Schweizer/Hafner (Fn. 72), Rn. 23 zu Art. 20.

87 Ulrich Häfelin/Walter Haller/Helen Keller/ Daniela Thurnherr, Schweizerisches Bundesstaatsrecht, 9. Aufl., Zürich 2016, Rn. 597.

88 Diese Spannung bestand seit jeher, dazu: Sundara Rajan, Copyright and creative freedom: a study of post-socialist law reform, London 2014, S. 26. Vgl. Hartmann (Fn. 16), Opt-in, S. 45. 


\section{b) Urheberrecht und damit assoziierte Fragen}

Im Vergleich zu anderen Rechtsordnungen 89 ermöglicht das Schweizer URG eine flexibel handhabbare Reaktion auf die informationstechnische Entwicklung.90 Erleichtert wird dies durch die Ausgestaltung der Nutzungsrechte als Generalklausel,91 verbunden mit Regelbeispielen.92 Doch ermöglichen sie auch eine Verpflichtung wissenschaftlicher Angestellter zu OA?93 Urheberrechtlich scheint Einigkeit zu bestehen, dass einer Universität nicht ohne Weiteres die Rechte am Werk der an ihr angestellten Wissenschaftlerinnen zufallen.94 Eine Publikation eines Werks in einem universitären Repositorium setzt mithin ei-

$89 \overline{\text { Siehe zu Beispielen, insbesondere aus dem deut- }}$ schen UrhG: Herbert Burkert/Peter Hettich/ Florent Thouvenin/Rehana Harasgama, Remembering and Forgetting in the Digital Age - A Position Paper, in: Information Research 2016, S. 60; ebenfalls Thomas Dreier, Urheberrecht auf dem Weg zur Informationsgesellschaft, Anpassung des Urheberrechts an die Bedürfnisse der Informationsgesellschaft, in: GRUR 1997, S. 859 ff., S. 863.

90 Siehe dazu Burkert/Hettich/Thouvenin/Harasgama (Fn. 89), S. 60, S. 57.

91 Art. 10 Abs. 1 URG.

92 Art. 10 Abs. 2 URG.

93 Aus arbeitsrechtlicher Sicht wird befürchtet, dass bei Bestehen einer solchen Verpflichtung, Wissenschaftler für Verlage unattraktiv würden, wenngleich eine solche Verpflichtung vertragsrechtlich für möglich gehalten wird, Hilty/Seemann (Fn. 11), S. 66; siehe in diesem Zusammenhang auch den polarisierenden sog. Heidelberger Appell.

94 Dazu umfassend Brigitte Bieler, Die Übertragung des Urheberrechts, in Konstellationen des Arbeits-, Auftrags-, Werkvertrags- und Produzentenverhältnisses sowie in ausgewählten Bereichen des Umstrukturierungsrechts, Basler Studien zur Rechtswissenschaft, Privatrecht, Band 117, Basel 2014. Aus deutscher Sicht dazu auch Joachim Dorschel, Open Access und Urheberrecht: Open Source in neuem Gewand?, in: Hagenhoff, S. 235 ff., S. 240 ff. Zur Frage des Zufallens von Urheberrechten in Arbeitsverhältnissen, auch mit Fokus auf den Wissenschaftsbetrieb: Manfred Rehbinder, Schweizerisches Urheberrecht, 3. Aufl., Bern 2000, Rn. 175 ff. ne explizite Rechteeinräumung voraus. 95 Dies ergibt sich zum einen aus dem Schöpferprinzip des Art. 6 URG, ${ }^{66}$ sowie aus Art. 9 Abs. 2 URG, 97 wonach der Urheber darüber entscheiden kann, was er wie, wo und wann veröffentlichen möchte. Entscheidet er sich im Rahmen seiner Vertragsautonomie, mit dem Verlag zu verhandeln, kommt ihm in der Praxis meist eine schwächere Verhandlungsposition als dem Verlag zu. ${ }^{8}$ Infolgedessen treten Urheber oftmals auch, z.T. unwissentlich, die entsprechenden «OnlineRechte» ab. 99 Gleichwohl könnten relativ wirkende, einfache Lizenzen ${ }^{100}$ die $\mathrm{Zu}$ rückbehaltung problemlos ermöglichen. ${ }^{101}$ Vorlagen für solche Lizenzen bie-

$95 \overline{\text { Die Universität kann somit nur derivativ Rechte }}$ erwerben, Hilty/Seemann (Fn. 11), S. 25; Hilty, Urheberrecht, Bern 2011, Rn. 314, 316.

96 Statt vieler: Denis Barrelet/Willi Egloff, Le nouveau droit d'auteur, Commentaire de la loi fédérale sur le droit d'auteur et les droits voisins, 3. Aufl., Bern 2008, S. 34 f.; Hilty/Seemann (Fn. 11), S. 24.

97 Barrelet/Egloff (Fn. 96), S. 49 f.: Droit de divulgation nach Art. 9 Abs. 2 URG.

98 Für eine Stärkung der schwächeren Partei durch zwingende Vertragsrechtsbestimmungen, wie etwa im Miet- oder Arbeitsrecht plädierend Hilty (Fn. 95), Urheberrecht, Rn. 38; gleichwohl soll nicht unerwähnt bleiben, dass die grössten Schweizer Verlage, den Autoren mit relativ kurzen Sperrfristen entgegenkommen, etwa bei Schulthess 3 Monate, bei Dike 6 Monate, sowie bei Stämpfli und Helbing gemäss Art. 382 Abs. 3 OR jeweils 3 Monate. Dazu Bernhard Dengg, Slide 24.

99 Hilty/Seemann (Fn. 11), S. 34 ff., bezeichnen damit das Recht zum Upload (Vervielfältigungsrecht i.S.d Art. 10 Abs. 2 lit. a URG), sowie der Zugänglichmachung (Art. 10 Abs. 2 lit. c URG).

100 Im Gegensatz zur absoluten Übertragung der Rechte, Hilty/Seemann (Fn. 11), S. 31 ff. Zum zulässigen Umfang der Übertragung von Urheberrechten siehe Bieler (Fn. 94), S. 45 ff., insb. S. $65 \mathrm{ff}$.

${ }_{101}$ Margo Bargheer, Open Access und Universitätsverlage: Auswege aus der Publication Crisis? in: Hagenhoff (Fn. 94), S. 173 ff., S. 181. Gegenüber den Repositorien sollte er auf seinen urheberrechtlichen Vergütungsanspruch verzichten, dazu Dorschel (Fn. 94), S. 246 ff. 
tet etwa SPARC. ${ }^{102}$ Diese können dann in einen entsprechenden Vertrag integriert werden. ${ }^{103}$

Ohne Vertragsverhandlungen und Vereinbarungen greift der Gesetzeswortlaut. ${ }^{104}$ Dabei ist, wenn auf den Verlagsvertrag schweizerisches Recht anwendbar ist, insbesondere Art. 381 Abs. 1 OR $\mathrm{zu}$ beachten, wonach nur diejenigen Rechte übertragen werden, derer es für die Durchführung des Vertrages tatsächlich bedarf. ${ }^{105}$ Schliesst der Urheber einen Verlagsvertrag mit einem OA-Verlag oder Repositorium, stehen in der Regel nicht mehr Vervielfältigungs-106 und $\mathrm{Zu}-$ gangsrechte ${ }^{107}$ im Vordergrund, sondern die technischen und vertrieblichen Dienstleistungen des Anbieters. ${ }^{108}$ Das Interesse des Urhebers an der Gewährung einer einfachen Lizenz entspricht im Ergebnis auch dem der Universität als

$102 \overline{\text { The Scholarly Publishing and Academic Re- }}$ sources Coalition.

103 Hilty/Seemann (Fn. 11), S. 28.

104 Wobei zu beachten ist, dass die Art. 380 ff. OR nicht zwingend sind und mithin Standardverträge und Usanzen überwiegen. Dazu Peter Breitschmid, Zwölfter Titel: Der Verlagsvertrag, in: Jolanta Kren Kostkiewicz/Stephan Wolf/Marc Amstutzu/Roland Fankhauser, OR Kommentar Schweizerisches Obligationenrecht, 3. Auflage, Zürich 2016, S. 1080.

105 Sog. Zweckübertragungstheorie, dazu Reto M. Hilty/Reto Arpagaus, Bundesgesetz gegen den unlauteren Wettbewerb (UWG), Basler Kommentar, Basel 2013, Art. 381 OR, Rn. 4; Rehbinder (Fn. 94), Rn. 165. Aus deutscher Sicht wiederum: Dorschel (Fn. 94), S.238 f. Man beachte, dass die Abs. 2 und 3, welche weitgehende Ausnahmen vorsehen, nicht auf Online Publikationen übertragbar sind, so jedenfalls Hilty/Seemann (Fn. 11), S. 38 ff.

106 I.S.v. Art. 10 Abs. 2 lit. a URG.

107 Art. 10 Abs. 2 lit. c URG.

108 Dorschel (Fn. 94), S. 235 ff., S. 238. Zum Verlagsvertrag im Lichte der technischen Entwicklungen und $\mathrm{OA}$ im Verlagsvertragsrecht: Inge Hochreutener, Der Verlagsvertrag: Art. 380-393 OR, Überarb. Diss. Fribourg 2010, in: Jörg Schmid, Kommentar zum schweizerischen Zivilrecht, S. 12 ff., sowie S. 154 ff.
Betreiberin des Repositoriums. Letztere hat im Idealfall kein Interesse an einer umfassenden oder gar kommerziellen Rechteverwertung. ${ }^{109}$ Aus diesem Grund ergeben sich Probleme insbesondere dann, wenn der Urheber von vornherein durch einen Verlagsvertrag ${ }^{110}$ gebunden ist. In diesem Fall erscheinen Verhandlungen zwischen der Universität und dem Verlag als am vielversprechendsten für alle Beteiligten. ${ }^{111}$ So wie z.B. in den USA, ${ }^{112}$ können kooperierende Verlage öffentlichkeits- und werbewirksam auf der Universitätswebsite hervorgehoben werden. ${ }^{113}$

21 Insgesamt ist festzustellen, dass das geltende schweizerische URG OA-Publikation zwar ermöglicht, aber nicht fördert. Welche Änderungen zugunsten OA durch den Gesetzgeber oder die Universitäten wären denkbar? Zum einen wird eine Kombination öffentlich-rechtlicher sowie urhebervertragsrechtlicher Regelungen diskutiert. ${ }^{114}$ Per zwingender gesetzlicher Anordnung verbliebe dem Urheber gemäss diesem Vorschlag das einfache Recht zur öffentlichen Zugäng-

109 Dorschel (Fn. 94), S.235 ff.

${ }_{110}$ Der Verlagsvertrag als einziger gesetzlich geregelter Urheberrechtsverwertungsvertrag: Breitschmid (Fn. 104), S. 1080 ff. Ebenfalls umfassend zum Verlagsvertrag: Hochreutener (Fn. 33).

111 Hilty/Seemann (Fn. 11), S. 67 ff.

112 Etwa an der Universität Harvard; siehe Kap. III.1.

113 In der Schweiz etwa an der Universität Basel.

114 Man beachte dazu die Diskussion im Vorlauf der Einfügung des $§ 38$ Abs. 4 UrhG in Deutschland: etwa bei Gerd Hansen, Zugang zu wissenschaftlicher Information - alternative urheberrechtliche Ansätze, GRUR Int 2005, S. 378 ff., 386 ff.; ebenfalls Dorschel (Fn. 94), S. 242. Für die Schweiz ist hier insb. die Vernehmlassungsantwort des Center for Information Technology, Society, and Law (ITSL) der Universität Zürich, verfasst von Florent Thouvenin und Daniel Hürlimann, zu vergleichen. Darin wird eine Änderung im Verlagsvertragsrecht zugunsten der Autorinnen vorgeschlagen. 
lichmachung. Sofort, oder nach Ablauf einer Sperrfrist, könnte oder müsste er die Werke in ein Repositorium hinterlegen ${ }^{115}$ Der weitere Vorschlag, Zwangslizenzen einzuführen, erscheint als Relikt eines früheren Urheberrechts und mithin nur bedingt zielführend. ${ }^{116}$ Schrankenregelungen ${ }^{117}$ zugunsten des Repositoriumbetreibers sind insbesondere am Dreistufentest ${ }^{118}$ zu messen und erweisen sich jedenfalls als politisch schwer durchsetzbar, weil sie dem Urheber von vornherein einen Teil seines Urheberrechts entziehen würden. ${ }^{119} \mathrm{Zu}$ beachten ist, dass die meisten dieser Vorschläge keine OA-Verpflichtung enthalten, sondern den OA-Gedanken anderweitig fördern. Vorschläge für OA-Verpflichtungen betreffen in der Regel nur das Hochschulrecht, sowie jenes von Förderorganisationen wie dem Schweizerischen Nationalfonds (SNF).

\section{c) Wettbewerbsrecht}

Einzelnen Anbietern renommierter Fachjournalen fällt eine quasi-monopolistische Stellung zu. Diese wird durch verschiedene Trends noch verstärkt.120 Wettbewerbsrechtliche Aspekte sind zudem bei Online-Plattformen mit einem festen Kundenkreis festzustellen. Bieten

115 Vergleichbar mit dem deutschen Modell. Dabei dürfen jedoch die daraus resultierenden Folgeprobleme keinesfalls unbeachtet bleiben. Siehe Kap. III.3.

116 Roland von Büren/Lucas David, Schweizerisches Immaterialgüter- und Wettbewerbsrecht, 3. Aufl., Basel 2014, S. 179; Dorschel (Fn. 94), S. 242; Reto $M$. Hilty, Renaissance der Zwangslizenzen im Urheberrecht? in: GRUR 2009, S. 633 ff.; Hilty/Seemann (Fn. 11), S. 96.

117 I.S.v. wissenschaftlichen Publikationen ohne Zustimmung des Rechtsinhabers allgemein zugänglich zu machen.

118 Art. 9 Abs. 2 RBÜ; Art. 13 TRIPS; Art. 10 WCT.

119 Dorschel (Fn. 94), S. 245 ff.; Hilty (Fn. 95), S. 179, 189; Hilty/Seemann (Fn. 11), S. 92 ff.

120 Hartmann (Fn. 9), Agenda, S. 35. sie für Kunden einen Mehrwert, erschweren sie zukünftigen Konkurrenten den Markteintritt. ${ }^{121}$ Individuell kann diese Situation durch Selbstarchivierung zum Teil vermieden werden. Kollektiv und nachhaltig durch die Schaffung und Bewahrung von OA-Geschäftsmodellen. ${ }^{122}$

23 Bei der Förderung von Open Access wird regelmässig die kartellrechtliche Preiskontrolle gegenüber den grossen Verlagskonzernen, und damit eine Massnahme zum Erhalt des Wettbewerbs, als Ansatz genannt. ${ }^{123}$ Wird nun, im Rahmen der nationalen OA-Agenda, eine Verpflichtung von Wissenschaftlern zur OAPublikation ihrer Werke in institutionellen Repositorien angestrebt, so erscheinen auch diese Repositorien als weitere Akteure im Wettbewerb, denn sie bilden faktisch einen Teil der Verlagsbranche und vermögen mitunter als potentielle Konkurrenz aufzutreten.124

\section{Das Beispiel der Universität St. Gallen und des Nationalfonds}

24 Der Wissenschaft ist Selbstregulierung immanent. Sie bestimmt die Formen für Publikation, Kommunikation und Rezeption. ${ }^{125}$ Infolgedessen haben auch in der Schweiz zahlreiche Institutionen bereits OA-Server, respektive -Archive, geschaffen. ${ }^{126}$

121 Hartmann (Fn. 9), Agenda, S. 35.

122 Zum Ganzen: Max Planck Digital Library Open Access Policy White Paper: Disrupting the subscription journals' business model for the necessary large-scale transformation to open access.

123 Hamann (Fn. 7), S. 99. Genannte Alternativen dazu bilden die Urheberrechtsschranke (Vorbild England) und das Zweitveröffentlichungsrecht (Vorbild Deutschland).

124 Hilty/Seemann (Fn. 11), S. 20. Massgebend sind damit insb. KG und UWG. Allg. zur Konkurrenz in der Verlagsbranche: Lessig (Fn. 35), S. 8 f.

125 Hartmann (Fn. 9), Agenda, S. 28.

${ }^{126}$ Eine Auflistung verschiedener institutioneller 
Von der technischen Umsetzung getrennt zu betrachten sind die verschiedenen OA-Strategien der Universitäten. Diese unterscheiden sich hinsichtlich Form, Inhalt und institutioneller Einbettung. ${ }^{127}$ An der Universität St. Gallen besteht z.B. ein OA-Reglement, das verschiedene Aspekte regelt. Die Pflichten der Forschenden umfassen die Bereitstellung bibliographischer Daten, von Volltexten als pre- oder post-print-Version, sowie die Sicherstellung von nicht ausschliesslichen Verwertungsrechten in Verlagsverträgen. Ein höheres Mass an Verbindlichkeit ergibt sich mit den Ausführungsbestimmungen zur Promotionsordnung für das Doktorat vom 2. Mai 2017. Demnach haben die Doktorierenden gemäss Art. 31 Abs. 1 zu erklären, «dass sie der Universität St. Gallen die Rechte übertragen, die Dissertation elektronisch zu speichern, sie in Datennetzen öffentlich zugänglich zu machen sowie bei Bedarf weitere Druckexemplare für die Professorenschaft der Universität St. Gallen herzustellen.»

Allgemein ist in der Schweiz insbesondere der Schweizerische Nationalfonds (SNF) mit Verpflichtungsforderungen aufgefallen. Inhaltlich soll die oben erwähnte Vision 2024 die Einführung und Abstimmung von OA-Politiken, Verhandlungen mit Verlagshäusern (sog. Offset Agreements, auch «Offsetting Way»), ebenso wie die Nutzung alternativer Publikationsformen, ${ }^{128}$ die Koordinierung

(u.a. universitären) Schweizer Archive findet sich unter OpenDOAR mit 18 Einträgen und über das Registry of Open Access Repositories ROAR mit 19 Einträgen.

127 Exemplarisch und zum Vergleich: ETHZ, als Policy; UNI-LU als Richtlinie; UZH, als Leitlinie.

${ }^{128}$ Womit insb. direkt der «Gold Way» beschritten werden könnte. und Zusammenlegung von Ressourcen, ${ }^{129}$ die Bildung eines unterstützenden regulatorischen Rahmens, den Aufbau eines nationalen Monitoring-Systems, sowie die Kommunikation und Sensibilisierung im Allgemeinen ermöglichen. Dabei sind verschiedene Szenarien denkbar, welche von der Belassung der jetzigen Situation, über die Berücksichtigung der drei Grundoptionen,130 bis zu einer Kombination dieser reicht. ${ }^{131}$

27 Als Mitunterzeichner der Berliner Erklärung versteht sich der SNF als Förderer von OA-Bestrebungen und versucht den politischen Rahmen zu schaffen. Es überrascht somit nicht, dass von den Beitragsempfängerinnen die Zustimmung zur Open-Access-Veröffentlichung verlangt wird. ${ }^{132}$

\section{Herausforderungen für die Einfüh- rung von OA-Pflichten}

28 Die unterschiedlichen Interessen der beteiligten Akteure, mithin also die Institutionen, die einzelnen Forscher und die Vertreter der Verlagsbranche, ${ }^{133}$ sowie

129 Hier könnte insb. der «Green Way»-Ansatz optimiert und effizienter gestaltet werden, das durch die Arbeit mit bereits bestehenden (institutseigenen) Infrastrukturen.

130 «Offsetting Way», «Green Way», «Golden Way». Siehe Kap. III.2.

${ }^{131}$ Vgl. zum Ganzen: Nationale Open-AccessStrategie für die Schweiz, 5. Massnahmen, S. $4 \mathrm{f}$. In dieser Arbeit nicht angesprochen, aber doch wesentlicher Entscheidungsfaktor für oder gegen die einzelnen Szenarien sind die Kosten. Allein dazu liesse sich eine ganze Abhandlung widmen.

${ }^{132} \mathrm{Zu}$ den genauen Vorgaben des SNF in Bezug auf Open Access siehe auch das Ausführungsreglement zum Beitragsreglement, insb. S. $32 \mathrm{ff}$.

${ }_{133} \mathrm{Zu}$ den Interessen der Forschenden vgl. Urteil 1C_40/2017 vom 5. Juli.2017, E. 6.2.1 f. Diese Interessen müssen aber auch artikuliert werden. Dabei bildet insb. die adäquate Repräsentation eine Herausforderung. Zur Vertretung der Autoren durch die Universitäten und einzelnen Fakultäten: Duranceau/Kriegsman (Fn. 20), S. 92. 
der internationale Kontext der OABestrebungen ${ }^{134}$ machen die mögliche Einführung von OA-Pflichten zu einem komplexen Unterfangen. Dieser Umstand zeigt sich insbesondere dann, wenn eine funktionierende und bewährte Regelung von OA-Pflichten auf andere Institution übertragen werden soll. ${ }^{135}$ Auch für die Schweiz, mit einem dezentralisierten Bildungs- und Forschungssystem, ${ }^{136}$ ist dies kaum anders. ${ }^{137}$ Die rechtlich-institutionelle Verankerung von OAVerpflichtungen wäre dabei wichtig für die Umsetzung, aber kein alleiniger Erfolgsgarant. Vielmehr müssen auch die einzelnen Wissenschaftlerinnen von der Idee überzeugt sein.138 Überzeugung kann insbesondere dann entstehen, wenn mit OA-Publikationen ein vergleichbarer Reputationsgewinn verzeichnet werden kann. Begleitend ist seitens der Universitäten persönliche Überzeugungsarbeit bei Mitarbeitenden zu leisten. ${ }^{139}$ Wird bei

134 Vgl. Klara Keutel, Der goldene Weg zur freien Wissenschaft, FAZ vom 7. Mai 2016.

135 Duranceau/Kriegsman (Fn. 20), S. 77. Siehe Kap. III.1, 2: Hier war die Adaption der Verpflichtung nach Harvard-Vorbild auf zahlreiche weitere Institutionen erfolgreich, da auch hier die Fakultäten als treibende Kräfte auftraten.

${ }_{136}$ Siehe Kap. IV.

137 Nationale Open-Access-Strategie für die Schweiz, 3. Leitlinien, 1. Ein starker und einheitlicher Ansatz, S. 3. Nicht zu vergessen sind die Politiker resp. die politischen Interessen, die bei der Verpflichtungsfrage ebenso wie bei einer konkreten Satzung über Gewicht verfügen. Zur Rolle der Politik als Teil des Gemeinwesens in der Netzpolitik im Allgemeinen: siehe Dirk Heckmann, Herausforderungen für das Gemeinwesen 2.0, Freiheit und Fairness als Leitmotiv einer neuen Netzpolitik, wie sie in Deutschland diskutiert wird, in: digma 2011, S. $12 \mathrm{ff}$.

${ }_{138}$ Vgl. Priest (Fn. 10), S. 377; Duranceau/Kriegsman (Fn. 20), S. 77 ff.; Urteil 1C_40/2017 vom 5. Juli 2017 E. 6.2.1 f. Zum an sich OA-freundlichen Wissenschaftsbegriff siehe Kap. IV.1.a).

139 Müßig (Fn. 54), S. 227. Hier wird aber auch angeführt, dass momentan mitunter bestehende Reputationsunterschiede (in den Geistes- noch wesentlich stärker ausgeprägt als in den Naturwis- fremden Forschungsergebnissen oftmals ein weitreichender Zugang gefordert, werden eigene Arbeiten derzeit noch vornehmlich unter Verschluss gehalten. ${ }^{140}$

\section{Fazit}

29 Von OA als Vision ist die konkrete Umsetzung zu unterscheiden. ${ }^{141}$ Für die Beantwortung der Frage, ob in der Schweiz eine Verpflichtung von Wissenschaftlerinnen $\mathrm{zu}$ OA-Publikationen eingeführt werden kann, wären konkretere Rahmenbedingungen wünschenswert. So ist insbesondere zu klären, ob der schweizerische Gesetzgeber in der laufenden $\mathrm{Re}$ vision des Urheberrechtsgesetzes bereit ist, ein Zweitveröffentlichungsrecht für Wissenschaftlerinnen einzuführen. Weiter besteht hinsichtlich der nur spärlich vorhandenen Rechtsprechung zur Wissenschaftsfreiheit gemäss Art. $20 \mathrm{BV}$ Konkretisierungsbedarf im Hinblick auf die Frage, inwieweit eine OAVerpflichtung grundrechtskonform wäre. ${ }^{142}$

3o OA-Strategien und OA-Regelwerke beschlagen als mögliche Grundlagen einer OA-Verpflichtung nicht nur die rechtliche Sphäre, sondern darüber hinaus auch gesellschaftliche und politische Fragen. Gerade zur Innovationsförderung wäre der nachhaltige Austausch zwischen Wissenschaft, Gesellschaft, Wirtschaft und Technik gewinnbringend. ${ }^{143}$ Während die

senschaften) durch die vertiefte Etablierung von OA relativ zügig überwunden werden könnten.

140 Steinhauer (Fn. 7), S. 18 ff., 55 f.; vgl. SNF, Open Access to Publications.

${ }^{141}$ Siehe Kap. I, II, III.

${ }_{142}$ Siehe Kap. IV.1.a.

143 Siehe Kap. IV.3. Beispielhaft hierzu das Projekt «In-no-Futures» der Universitäten Bern und Neuchâtel. 
Befürworter in einer OA-Verpflichtung einen effektiven Weg zur Verwirklichung der OA-Vision sehen und damit eine zukunftsweisende Ausrichtung der Wissenschaft erblicken, ${ }^{144}$ befürchten Gegenstimmen eine Gefährdung des geltenden Rechtsverständnisses, des Verlagswesens, ebenso wie der Buchkultur. ${ }^{145}$

31 Vor diesem Hintergrund stellt sich die Frage, wie eine OA-Verpflichtung gegebenenfalls gesetzgeberisch ausgestaltet werden sollte: Als Zwang oder etwas abgeschwächt als grundsätzlich «verbindliche Option» mit Opt-Out-Möglichkeit?146 Hier werden insbesondere drei Vorschläge als plausibel betrachtet, die idealerweise kombiniert würden. Möglich ist eine hochschulrechtliche OA-Verpflichtung mit Opt-Out-Möglichkeit nach englischem Vorbild, 147 die Einführung eines Zweitveröffentlichungsrechts wie in der Bundesrepublik Deutschland, ${ }^{148}$ sowie eine kartellrechtliche149 Preiskontrolle hinsichtlich quasi-monopolistischer Verlagskonzerne. 150

$144 \overline{\text { Exemplarisch: Nationale Open-Access-Strategie }}$ für die Schweiz, 6. Implementierung, S. 6. Zur effektiven Verbreitung von Wissen, nach Wunsch der Forschenden: Shieber (Fn. 47), S. 5 ff.; Fischmann Afori (Fn. 17), S. 398 ff.

145 Insb. in Deutschland wurde diese Diskussion schon vertieft geführt. Siehe Kap. III.3. (beispielhaft: der Heidelberger Appell und die sehr zahlreichen Gegenstimmen [Fn. 98]).

146 Steinhauer (Fn. 7), S. 44. Diese können aus Förderrichtlinien (betroffen sind hier Projekte welche von nationaler Forschungsförderung profitieren) folgen oder aber direkt aus dem Hochschulrecht, dazu Hamann (Fn. 7), S. 99.

147 Vgl. Kap. III.2.

148 Siehe Kap. III.2.

149 Wenngleich aus kartellrechtlicher Sicht das Vorhandensein eines Monopols nur schwerlich zu begründen wäre.

${ }^{150}$ Hamann (Fn. 7), S. 99: In Bezugnahme auf Matthias Seemann. 